\title{
The head involution defective gene of Drosophila melanogaster functions in programmed cell death
}

\author{
Megan E. Grether, ${ }^{1}$ John M. Abrams, ${ }^{2}$ Julie Agapite, Kristin White, ${ }^{3}$ and Hermann Steller ${ }^{4}$ \\ Howard Hughes Medical Institute, Department of Brain and Cognitive Sciences, Department of Biology, Massachusetts \\ Institute of Technology, Cambridge, Massachusetts 02139 USA
}

\begin{abstract}
Deletions of chromosomal region, $75 \mathrm{C1}, 2$ block virtually all programmed cell death (PCD) in the Drosophila embryo. We have identified a gene previously in this interval, reaper (rpr), which encodes an important regulator of PCD. Here we report the isolation of a second gene in this region, head involution defective (hid), which plays a similar role in PCD. hid mutant embryos have decreased levels of cell death and contain extra cells in the head. We have cloned the hid gene and find that its expression is sufficient to induce PCD in cell death defective mutants. The hid gene appears to encode a novel 410-amino-acid protein, and its mRNA is expressed in regions of the embryo where cell death occurs. Ectopic expression of hid in the Drosophila retina results in eye ablation. This phenotype can be suppressed completely by expression of the anti-apoptotic p35 protein from baculovirus, indicating that $\mathbf{p} 35$ may act genetically downstream from hid.
\end{abstract}

[Key Words: Drosophila; programmed cell death; apoptosis]

Received April 6, 1995; revised version accepted June 12, 1995.

Cell deaths that occur during the development of essentially all metazoan animals display a characteristic ultrastructural morphology known as apoptosis (Kerr et al. 1972; Wyllie et al. 1980). Because it is thought that these natural cell deaths result from the execution of an active, gene-directed cell suicide program, the process of apoptosis is also referred to as programmed cell death (PCD). Strong support for this concept has come from genetic studies in the nematode Caenorhabditis elegans, where a large number of mutations affecting specific aspects of PCD have been isolated and ordered into a genetic pathway (for review, see Ellis et al. 1991; Hengartner and Horvitz 1994a,b). In particular, three genes, ced-3, ced-4, and ced-9, have been shown to control the onset of all somatic PCDs in the nematode. Interestingly, two of these genes, ced-3 and ced-9, have mammalian homologs that are believed to play a similar function during apoptosis. The ced-3 gene is homologous to a family of cysteine proteases that includes interleukin- $1 \beta$ converting enzyme, and ced-9 is a member of the $b c l-2$ family (Yuan et al. 1993; Hengartner and Horvitz 1994c; Wang et al. 1994). Moreover, expression of human Bcl-2 can suppress some PCD in C. elegans and can partially

Present addresses: ${ }^{1}$ University of California, San Francisco, Department of Biochemistry and Biophysics, San Francisco, California 94143-0448 USA; ${ }^{2}$ Department of Cell Biology and Neuroscience, University of Texas, Southwestern Medical Center, Dallas, Texas 75235-9039 USA, ${ }^{3}$ Cutaneous Biology Research Center, Massachusetts General Hospital, Harvard Medical School, Charlestown, Massachusetts 02129 USA.

${ }^{4}$ Corresponding author. substitute for the loss of ced-9 function (Vaux et al. 1992; Hengartner and Horvitz 1994c). Therefore, at least some components of the cell death pathway have been conserved throughout evolution. This idea is also supported by reports that the baculovirus $\mathrm{p} 35$ gene can inhibit PCD in insects, nematodes, and mammalian neurons (Rabizadeh et al. 1993; Clem and Miller 1994; Hay et al. 1994; Sugimoto et al. 1994). However, despite considerable progress in identifying cell death genes, the molecular basis of apoptosis remains unknown. In addition, it is not clear how the death program is only activated in those cells that should be eliminated. Recent work on PCD in Drosophila indicates that this system may offer powerful new approaches for advancing our understanding in these areas.

In Drosophila, as in vertebrates, the induction of cell death depends on a variety of distinct signals, such as hormones, diffusible survival factors, intercellular interactions, ionizing radiation, and other cellular damage (see e.g., Fischbach and Technau 1984; Meyertholen et al. 1987; Steller et al. 1987; Bryant 1988; Magrassi and Lawrence 1988; Kimura and Truman 1990; Wolff and Ready 1991; Campos et al. 1992; Truman et al. 1992; for review, see Steller and Grether 1994). Cell deaths in Drosophila display many of the morphological and biochemical hallmarks of mammalian apoptosis (Bryant 1988; Abrams et al. 1993; Robinow et al. 1994; White et al. 1994). A number of mutations have been isolated that affect PCD in specific cells or tissues (see, e.g., Fristrom 1969; Wolff and Ready 1991; Abrams 1993; Bonini et al. 
1993; Cheyette et al. 1994). More recently, a large fraction of the Drosophila genome has been systematically surveyed for genes that are required for PCD by analyzing the pattern of cell death in embryos homozygous for previously isolated chromosomal deletions (White et al. 1994). In this screen a single region on the third chromosome has been identified which is required for all PCDs that normally occur in the Drosophila embryo. Deletions in chromosomal region $75 \mathrm{Cl}, 2$, such as $\mathrm{Df}(3 \mathrm{~L}) \mathrm{H} 99$ (abbreviated H99), block virtually all PCD in response to several distinct death-inducing signals. Molecular analysis of this region has led to the identification of a gene, reaper $(r p r)$, which appears to play a central control function for the induction of apoptosis in Drosophila (White et al. 1994). Expression of $r p r$ is sufficient to restore PCD to H99 mutant embryos, and reaper mRNA appears to be specifically expressed in cells that are destined to die. However, these results did not exclude the possible existence of another, functionally redundant cell death gene in this interval. In this paper, we present evidence that a second gene in the $\mathrm{H} 99$ interval, head involution defective (hid), has a similar role in PCD as rpr. We find that hid mutants have reduced levels of apoptosis and show that expression of a hid cDNA under the control of the $h s p 70$ heat shock promoter is sufficient for the rapid and widespread induction of apoptosis in both wild-type and $\mathrm{H} 99$ mutant embryos. Furthermore, ectopic expression of hid in the developing retina results in eye ablation. This phenotype can be completely suppresed by coexpression of the baculovirus p35 protein, which has been shown previously to suppress apoptosis in multiple contexts (Rabizadeh et al. 1993; Sugimoto et al. 1994; Clem and Miller 1994; Hay et al. 1994). The ability of hid to kill cells is independent of $r p r$ function, and both genes appear to act at the same step in the cell death pathway. Interestingly, we find some limited amino acid similarity between the deduced polypeptide sequences of hid and rpr, suggesting that the overall functional similarities between both genes may extend to the molecular level. Our results indicate that hid and rpr represent parallel functions for the induction of PCD in Drosophila.

\section{Results \\ Genetic analysis of chromosomal region 75C1,2}

We have reported previously that the genomic region included in $\mathrm{H} 99$, corresponding to polytene chromosome bands $75 \mathrm{Cl}, 2$, is required for PCD (White et al. 1994). Embryos homozygous for H99 lack all PCD that is normally induced in response to various death-inducing signals. To identify single gene mutations in this interval we performed a standard $F_{2}$ chemical mutagenesis for mutations that produced either lethal or visible phenotypes in combination with the $\mathrm{H} 99$ deficiency (see Materials and methods). We isolated five alleles corresponding to only a single complementation group, and none of these alleles was fully cell-death defective. One possible explanation for our failure to isolate single-gene mutants lacking PCD within the $\mathrm{H} 99$ interval is the presence of multiple cell death genes with overlapping function. In this case, inactivation of any one gene would be insufficient to completely abolish apoptosis. All five alleles isolated in our screen failed to complement mutations in the hid locus identified previously (Abbott and Lengyel 1991) and displayed significantly reduced viability. hid mutants have a pronounced defect in the morphogenetic movements of head involution but otherwise develop an overall normally segmented cuticle and reach advanced embryonic stages (Abbott and Lengyel 1991). Because head involution is associated with extensive cell death, we considered the possibility that the hid phenotype results from decreased levels of PCD.

\section{hid mutant embryos have decreased levels of PCD and extra cells in the head region}

We analyzed the pattern of cell death in hid embryos using the TUNEL technique (Gavrieli et al. 1992). This method labels the nuclei of dying cells by allowing in situ detection of the DNA fragmentation that occurs during apoptosis. Because of the need to use parents heterozygous for the hid mutation, only $25 \%$ of all embryos will be homozygous mutant (see Materials and methods). Therefore, it was important to distinguish hid ${ }^{-}$ embryos from their siblings to unequivocally determine whether hid mutants have reduced levels of PCD. For this purpose, we combined the TUNEL labeling with an in situ hybridization protocol. Using an allele of hid, $h_{i d}{ }^{W R+X 1}$, which does not express hid mRNA (see below), we were able to identify hid - embryos based on their lack of an in situ hybridization signal (see Materials and methods). Wild-type control embryos used for comparison were taken through the same procedure, except that no hid hybridization probe was included, as the in situ hybridization signal can obscure the TUNEL labeling.

We found that there was less apoptosis in hid mutants when compared to wild type. There was a general decrease in TUNEL labeling throughout the hid embryos, and this phenotype was most noticeable in the head region prior to completion of head involution (Fig. 1). This result is consistent with the striking defects in head morphogenesis, which result, in part, from a failure of the dorsal fold to migrate anteriorly in hid mutants (Abbott and Lengyel 1991; M.E. Grether and H. Steller, in prep.). It is possible that these morphogenetic defects result from the decrease of PCD in this region.

We have reported previously that $\mathrm{H} 99$ embryos contain many additional cells in the nervous system (White et al. 1994). Using an antibody against Krüppel (Gaul et al. 1987), we found that hid mutant embryos have extra cells in the head region, in particular, extra larval photoreceptor cells (Fig. 2). Wild-type embryos have an average of $9.3 \mathrm{Krüppel}^{+}$cells per photoreceptor cluster ( $n=60$ clusters) and never $>13$ cells in a single cluster. In contrast, in hid mutants we found an average of 15 cells per cluster ( $n=156$ clusters), and occasionally as many as 20 or more cells in a single cluster $(n=17)$. This increase in cell number is consistent with the observation that 

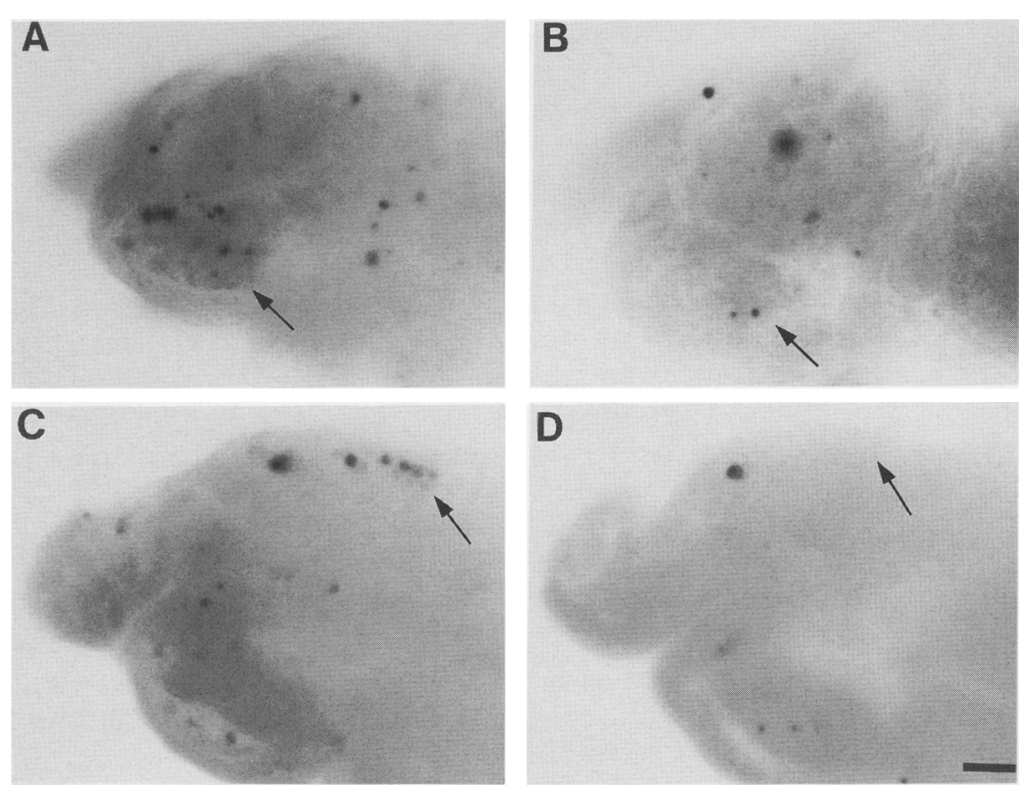

Figure 1. PCD in wild-type and hid mutant embryos. Lateral view of the heads of embryos labeled with TUNEL (see Materials and methods) to assay the relative levels of cell death in wild-type $(A, C)$ and $W R+X 1 / H 99(B, D)$ embryos. In this and all subsequent figures, anterior is to the left and dorsal to the top. $(A, B$ and $C, D)$ Equivalent focal planes in aged matched stage 12 embryos. In $A$, cell death predominates in the ventral head in the migrating gnathal segments. Little or no cell death is seen in this location in the hid embryo (B). Although cell death is found close to the site of dorsal fold migration in wild type $(C)$, cell death is not found in the corresponding head region in hid $(D)$. Overall, note how there is much less cell death in the hid mutant embryos $(B, D)$ than in the corresponding wild-type embryos $(A, C)$. Bar, 25 $\mu \mathrm{m}$. hid mutants had decreased amounts of cell death in the head region. In addition, we have also observed extra cells in the abdominal segments of hid mutants using an anti-engrailed antibody (Patel et al. 1989; data not shown). Taken together, these results indicate that hid function is required for the normal pattern of PCD in the Drosophila embryo.

\section{Molecular characterization of the hid locus}

We have speculated previously that the $\mathrm{H} 99$ interval may contain more than one functionally redundant cell death genes, one of which is rpr (Steller et al. 1994; White et al. 1994). The finding that hid has reduced levels of PCD is consistent with the idea that hid may function in the control of apoptosis. One prediction of this model is that hid expression should be sufficient to restore PCD to cell death defective H99 embryos. To test this prediction, we deceided to clone the hid gene. For this purpose, we extended the chromosomal walk of Segraves and Hogness (1991) to include all the DNA encompassed by the $\mathrm{H} 99$ deficiency and looked for the presence of transcripts derived from this region (see $\mathrm{Ma}$ terials and methods). In addition to $r p r$, we identified only three other transcription units that gave reproducible signals by Northern analysis. One of these corresponds to hid (see below). The embryonic expression pattern of the two other transcripts, as determined by in situ hybridization, showed no obvious correlation with the pattern of cell death in the Drosophila embryo. The unusually low density of transcripts in this region is consistent with the paucity of mutations producing a lethal or visible phenotype.

The molecular isolation of the hid locus was facilitated by the availability of a lethal P-element insertion, $1(3) 05014$, in the $75 \mathrm{Cl}, 2$ interval (Karpen and Spradling
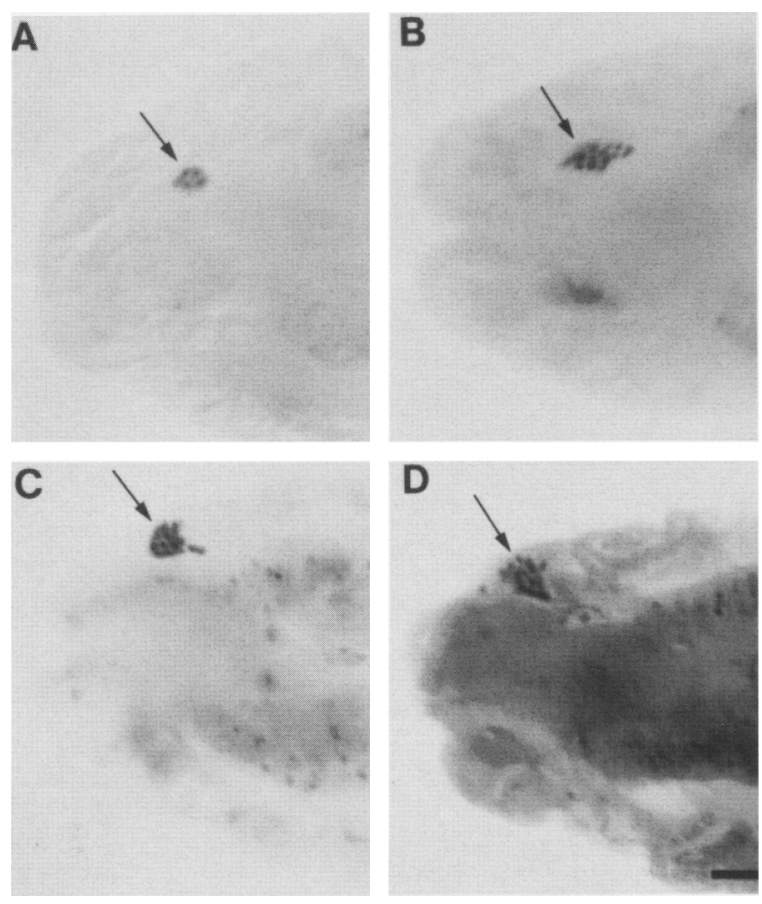

Figure 2. hid mutant embryos have extra cells in the larval photoreceptor organ. Dorsal view of embryos stained with antiKrüppel antibody to visualize the cells of the larval photoreceptor organ (Gaul et al. 1987). All embryos are of an equivalent stage. $(A)$ Wild-type embryo; $(B-D)$ the hid mutants $W R+X 1$, $A 206$, and $1(3) 05014 / H 99$, respectively. Arrows indicate those cells that will become the larval photoreceptors. Wild-type embryos have an average of 9.3 cells per photoreceptor cell cluster. In the embryo shown in $A$, five cells can be seen in this focal plane. hid mutants have an average of 15 cells per cluster. In the hid mutants $(B-D), 15,17$, and 15 cells are visible in each photoreceptor cell cluster, respectively. Bar, $25 \mu \mathrm{m}$. 

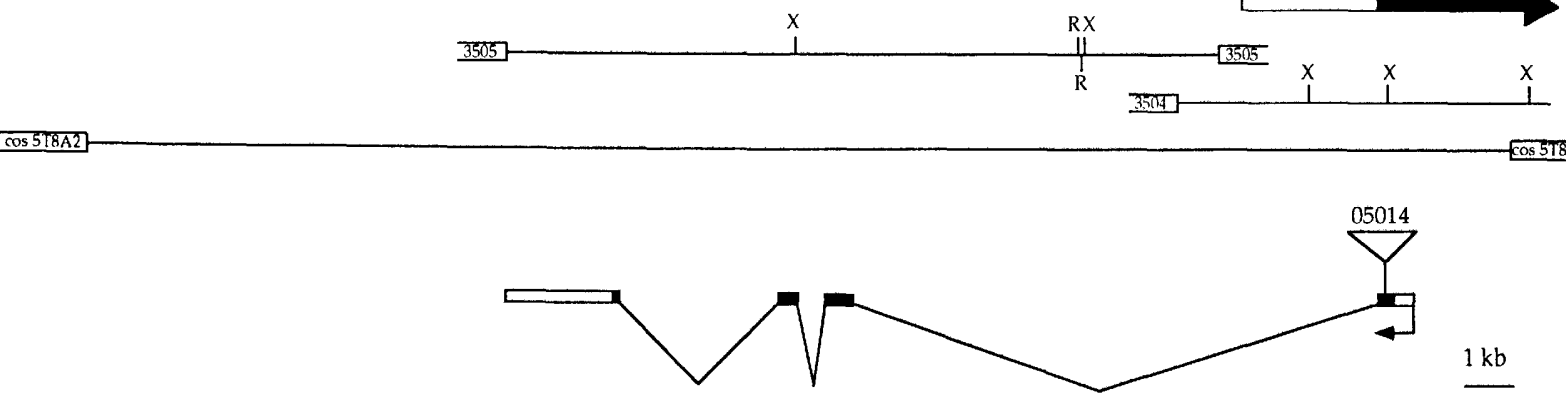

1 TGACAAAAAA TAAAAAACGAAATCCATCGTGAACAGTTTTGTGTTTTTAAATCAGTTCTAAACACGAAAAGGGTTGATGAAAAACGCAGAAGAATCCGAAAAACTAACTAACCGAGCAAA 120 121 AACTTGACTTGAGTGTTGTTTGACAAATCAGGAAAGATAAAAAACAAATCATAAGAAAAAACTGCACGAAAAATGAAAAAGTTTCTAATA TTCAAAATCTTGCACAAGAAATACAAAATC 240 241 AATTAA.AGTGAACTCTAACCAAAAGTTGTACACAAAATAAAAAGCAAAACAAAGCAGCGAAGAACAATCACAAGAAGAGCAAAGTGCCAACAAAGTGCAGGAAGGAAGGAAGCGGATAAG 360 361 GACAAAAAGGAAGCCAGCACACACACACACACCCACACAATGGCCGTGCCCTTTTATTTGCCCGAGGGCGGCGCCGATGACGTAGCGTCGAGTTCATCGGGAGCCTCGGGCARCTCCTCC 480 $\begin{array}{llllllllllllllllllllllllllllll}M & \text { A } & V & P & \text { F } & \text { Y } & \text { L } & \text { P } & \text { E } & \text { G } & \text { G } & \text { A } & \text { D } & \text { D } & \text { V } & \text { A } & \text { S } & \text { S } & \text { S } & \text { S } & \text { G } & \text { A } & \text { S } & \text { G } & \text { N } & \text { S } & \text { S } & 27\end{array}$

481 CCCCACAACCACCCACTTCCCTCGAGCGCATCCTCGTCCGTCTCCTCCTCGGGCGTGTCCTCGGCCTCCGCCTCCTCGGCCTCATCTTCGTCATCCGCATCGTCGGACGGCGCCAGCAGC 600

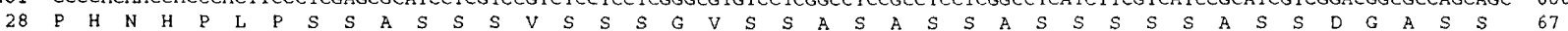
Y $\Downarrow$

721 CAGCAGAGTGCCCCGCAAATCTTCCAGTATCCGCCGCCAAGCCCCTCTTGCAATTTCACTGGCGGCGATGTGTTCTTTCCGCACGGCCATCCGAATCCGAACTCGAATCCCCATCCGCGC 840

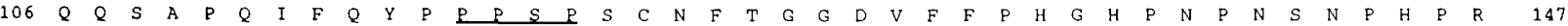

841 ACCCCCCGAACCAGCGTGAGCTTCTCCTCCGGCGAGGAGTACAACTTCTTCCGGCAGCAGCAGCCGCAACCACATCCGTCATATCCGGCGCCATCAACACCGCAGCCAATGCCACCGCAG 960

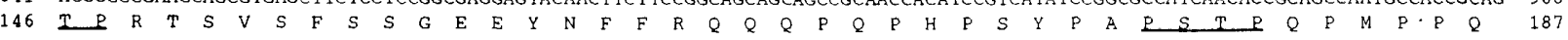

961 TCAGCGCCGCCGATGCACTGCAGCCACAGCTACCCGCAGCAGTCGGCGCACATGATGCCACACCATTCCGCTCCCTTCGGAATGGGCGGTACCTACTACGCCGGCTACACGCCACCACCC 1080

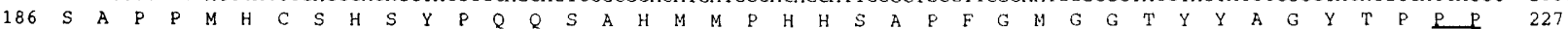

A206: T

1081 ACTCCGAACACGGCCAGTGCGGGCACCTCCAGCTCATCGGCGGCCTTCGGCTGGCACGGCCACCCCCACAGCCCCTTCACGTCGACCTCCACGCCGTTATCGGCGCCAGTGGCGCCCAAG 1200

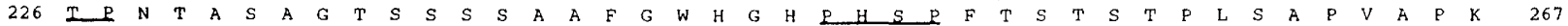
A206: T $\Downarrow$ A329: A

1201 ATGCGCCTGCAGCGCAGCCAGTCGGATGCGGCCAGACGCAAGCGATTGACCTCGACGGGCGAGGATGAGCGCGAGTACCAGAGCGATCATGAGGCCACTTGGGACGAGTTTGGCGATCGC 1320

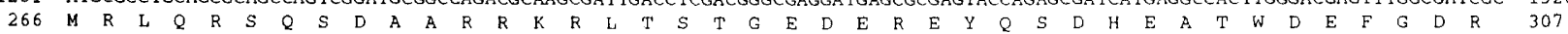

1321 TACGACAACTTTACGGCCGGCGGGAGCGTCTGCAGGAGTTCAATGGACGCATCCCGCCCCGGAAGAAGAAGAGCTCCAATAGCCACTCGAGCAGCAGCAATAATCCAGTCTGCCATACC 1440

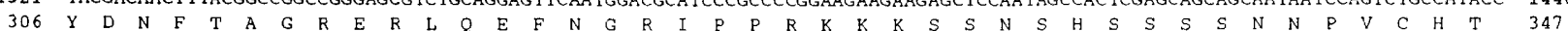

$\downarrow$

1441 GACAGCCAGTCCGGTGGTACATCCCAAGCGGAGAGCGGTGCCATCCATGGCCACATCAGTCAGCAGCGACAGGTGGAGCGAGAACGACAAAAGGCGAAGGCCGAGAAGAAGAAACCACAG 1560

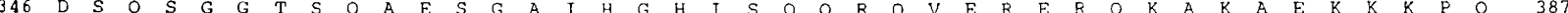

1561 AGCTTCACTTGGCCAATGTTGTGACCGTTTTCGTTTTGGCCATGGGCTGTGGCTTCTTTGCGGCGCGATGAAAGCGCAGGAGACGTGTAATCGAATGATCTA TAGTGAAATCAGCTAGC 1680

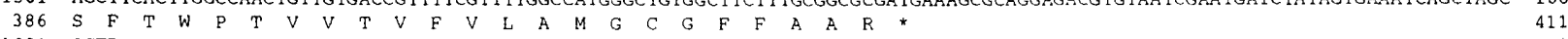
1681 CCTTAAGATATATGCCGATCTAAACATAGTTGTAGTTAAACCGTACATAAGTGCAACGAATTTATTGAACTGCAGGAGCGA.A.GCAGAAAGTCATTAATTCGTAAACGGATTGTTAGATA 1800 1801 CACAAACAGCCAACATACACGAAGAGTGTGCCTAAGATTAAGAAGGTTGACGGGACACAAGA.ACAATATATTCTATCTGTCTATGGTAACTGCATTTGTATTTCTAAAACGAAACGAAAG 1920 1921 ATAACAATCTTAACTGCTCAAAGTAATGAAAACTCTTAGACTGGCAAGAGACTCAAATCACACTTATTTTTTTGCTGATCCATATTTTTGTACAACCTTTTTAGCGATA.TTTACAAATTA 2040 2041 TACTAGTACAAAAAAAAGAGAGAGAGAGATAAGCAAAA 2041 TACTAGACAAAAAAAAGAGAGAGAGAGATAAGCAAAAGAAAACTGCCACTTTGAGATACTTTTGATAATCITGGTTTGCATTAATCATTCCACACTMCATTTTTATAACAAC 2160 2161 AAACAAAATACTCCAT

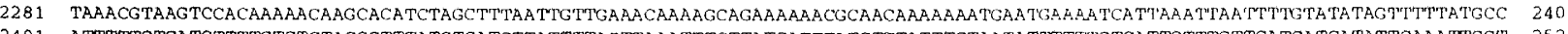
2401 ATTT'TTGTGATGTTTTGTGTCTACGGTTTATGTCATGTTATTTTAGTTAAAT'TTCTTA TGATTTA TGTTTATTTGTAATA TITTTYGTCATTGTTTGTTCATCATCATATTCAAATTGG' 2520 2521 CTCACA.TATAATAGTTTTAAGCTCCACGCCCGGGAGATTGATGGCAAAACGATTGAAATTTGGCCAGAAGP.GAGATAGTTTTCCCCATTCGTACACAGTCTT'TTTGGAATGCACA TTA 2640 2641 ATGATCTCTCACAATGGAAATTAA TGAAAATTGATCTCCGCAGCTAGCCAAAGTTAAAAAAGAAATGAAGAGGAAAACATATTCTA.TAGGCAATTTTCACTATATGCTAGAATTTCCCGG 2760 2761 GCGTTTCAATGCTAATCGAATACAGTGACATGAAACAAACATAGCGAAATPTTAAGAAAATCAATCAAAAAGAAAGAAAMACCAATTCCCAAAAATCGCATTGATCTCATGGATTTAT 2880 2881 ACAATACAATTACATCAACCGTTTTTTTACA.TGAGAAATGTTATAAAAAGCAGAAAGTGAAACACAGAAACATAAACAAAAATTAACGAAAAGCTTAGATATAAGTTCGCCAAGCGTTT 3000

3001 TAGTTCTATTTTCTAGA.ATGTCTAAGTCGGTTTAGTGAGTTTATTAAGCTGTCTTCGGACACAAGTTTATTTGTATATAAGCAATATTA TTTKGTGAGCCTAAGTGACAGTCCCAATCAA 3120

3121 ATCCAATCCAATATCACCCAGTCCGGACATTTCCCAGCAAAACAATAGACTATTCTCGCGTTCACATGTATCAATCTTAATTTGAATWACCACAAAATGAAATGA.AATACTAAAACCAT 3240

3241 ACACAAATGAAAAATTATTTTTGTAAATTGTTTGCATCA.AGTGAGCAAGGGGATTAGATTAAGGAATCATCCTTGCTTTATCCCCTGCTTATTGCTAATTAGTTTTCACAATGATCTCGG 33 60

3361 TAAAGTTTTGTGGCCTTGCGCCCAAAAGTCGTACAGATTTTTGGTTTGCCATAAATACTCGAACA.AAAAGTTPA TGAAAAACGAAGCAAA TGGAAAAAAA.ATCAGAATGA.A.AACA.AGAA 3480

3481 ATTTATATTWTTTCACCCAATGCTACTTAATCCGTTTTUG

3481 ATTWATAT 3601 T600

3601 TTTGGTGCCAAAACAGTGACAGATAATTAAATGTCTATAACCCGTGTATTCGCATATTATACATTTATACATATATCGTAACTTCAATGATAAGTTTGATTCTGAAATTTTGTCAACT 3720

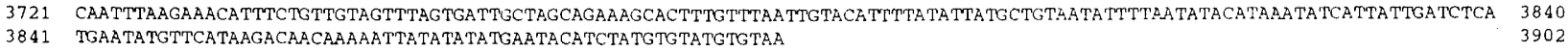

Figure 3. (See following page for $\mathrm{C}$ and legend. 
1992; Berkeley Drosophila Genome Project, pers. comm.). We found that $1(3) 05014$ fails to complement hid. To determine whether the P-element insertion causes the hid mutation, we mobilized the $05014 \mathrm{P}$ element (see Materials and methods). We obtained many independent reversion events, indicating that the P-element insertion is responsible for the hid phenotype. DNA flanking the 05014 P-element insertion was cloned by plasmid rescue and mapped with respect to the chromosomal walk (see Materials and methods). These sequences hybridized to a single transcript of $\sim 4.2 \mathrm{~kb}$ on Northern blots containing embryonic RNA. Several cDNA clones corresponding to this transcript were isolated from an eye disc cDNA library. The longest clone, $5 \mathrm{Al}$, had an insert of $3.9 \mathrm{~kb}$ and contained an open reading frame (ORF) of 410 amino acids (see below). This clone was used for all further analyses.

The 5Al cDNA was mapped to genomic clones in the interval to determine the genomic structure of this locus in wild-type and several hid alleles (Fig. 3A). A number of mutant alleles had chromosomal rearrangements that either broke within or deleted this transcription unit (Fig. 3A). One of these mutations, hid ${ }^{W R+X 1}$, deletes the $5^{\prime}$ end of this gene and completely abolishes its transcript, as determined by in situ hybridization. This allowed us to identify hid mutant embryos in the aforementioned TUNEL experiments. We also sequenced the coding region and intron/exon boundaries of several independently isolated hid alleles (Table 1; Fig. 3B). This analysis revealed that the $05014 \mathrm{P}$ element has inserted into the ORF between amino acids 105 and 106, thereby disrupting the putative coding sequence. No detectable P-element sequences remained in two independent reversion chromosomes that had been generated by mobilization of the 05014 P element, restoring the ORF in the $5 \mathrm{~A} 1$ transcript. In contrast, two chromosomes where the $05014 \mathrm{P}$ element had excised without restoring hid function had residual insertions of 38 and $40 \mathrm{bp}$ of P-element DNA that failed to restore the 5A1 ORF (Table 1). Finally, five of eight chemically induced alleles were associated with nucleotide changes that either introduced premature stop codons, missense mutations, or mutated a splice donor sequence (see Table 1). Taken together, these results demonstrate that the $5 \mathrm{Al}$ transcript is es- sential for $\mathrm{hid}^{+}$function. Therefore, we refer to it from now on as the 5Al hid cDNA.

The sequence of the $5 \mathrm{Al}$ cDNA is presented in Figure 3B. The longest ORF predicts a translation product of 410 amino acids with a deduced relative molecular mass of $43 \mathrm{kD}$. There are multiple translational stops in all three reading frames upstream of the relevant AUG start codon. The carboxy-terminal half of the predicted protein is fairly rich in charged residues, and the protein as a whole is relatively basic (predicted $\mathrm{pI}=8.27$ ). Hydropathy analysis shows that the predicted protein is fairly hydrophilic, with no hydrophobic regions of sufficient length to serve as either signal sequences or transmembrane domains. Analysis using BLAST and FASTA sequence comparison algorithms revealed no significant similarities with nucleotide or amino acid sequences reported previously. hid does, however, have limited sequence similarity to the rpr gene (Fig. $3 \mathrm{C}$ ). This is most striking in the first 11 amino acids, 6 of which are identical and 4 of which are conserved substitutions between hid and rpr. Also, in this block of amino acids there is the highest degree of identity between the rpr genes of Drosophila melanogaster and a distantly related species, Drosophila virilis (M.E. Grether, R. Jespersen, and H. Steller, unpubl.).

\section{Expression of hid is sufficient to induce cell death in H99 embryos}

To determine whether hid functions in PCD, we wanted to examine whether hid expression is capable of restoring apoptosis in $H 99$ embryos, which are deficient for both hid and rpr. Initially, we used a genomic cosmid clone containing the transcribed region of hid to generate germ-line transformants but failed to rescue hid mutants (data not shown). Presumably, this cosmid lacked 5 ' sequences required for hid expression. Unfortunately, the large size of the hid transcription unit (Fig. 3A) prohibits the use of cosmids that contain additional regulatory sequences and makes it technically impractical to use genomic DNA for rescue experiments. Therefore, we decided to examine the consequences of hid cDNA expression on PCD. For this purpose, we generated germ-

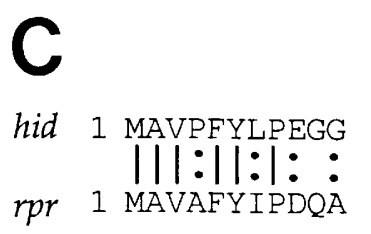

Figure 3. Molecular characterization of the hid locus. (A) Genomic map of the hid transcript. Clones 3505 and 3504 are genomic phages isolated by Segraves and Hogness (1990). The intron/exon structure of the hid transcript was determined by Southern blot analysis and DNA sequencing (see Materials and methods|. In the transcript, solid bars represent coding exons, open bars, untranslated sequences. The site of the 05014 insertion and direction of transcription are also shown. Large arrows above the genomic map represent genomic deficiencies in $75 \mathrm{Cl}, 2$ which extend in the direction shown by the arrows. All deficiencies either break within or delete this transcript entirely. In $W R+X 1$, the open portion of the arrow marks the genomic region in which the distal breakpoint must lie. (R) EcoRI; (X) XhoI. (B) Sequence of hid cDNA 5A1. The deduced amino acid sequence of the longest potential ORF is shown beneath the nucleotide sequence. Two upstream ATGs are underlined: Five potential MAP kinase consensus phosphorylation sites are underlined beneath the amino acid sequence. Intron positions are marked by open arrows. The site of the 05014 insertion is marked with an arrow. The polymorphisms identified in the sequenced hid alleles are shown in uppercase letters next to the name of each allele, above the cDNA sequence. (C) Comparison between the first 11 amino acids of hid and rpr. The predicted protein sequences of hid and rpr have some limited sequence similarity at their amino termini that is not shared with any other proteins in the data base. Bars indicate identities; double dots indicate conserved amino acid substitutions. 
Table 1. Molecularly characterized his alleles

Allele

1) $l(3) 05014$ P-element insertion at nucleotide 714, between amino acids 105 and 106

2) $40 \mathrm{C}$ imprecise excision of 1(3)05014

3) $8 D \quad$ imprecise excision of $1(3) 05014$

\begin{tabular}{|c|c|c|c|}
\hline Canton-S & 698 & GAGTGGGTCAGGATGTAC & CAGAGCCAGC \\
\hline $40 C$ & 698 & GAGTGGGTCAGGATGTACCATGATGAAATAACAT & T TTATTTCATCATGGGATGTACCAGAGCCAGC \\
\hline $8 D$ & 698 & GAGTGGGTCAGGATGTACCATGATGAAATAACATA & TGTTATTTCATCATGGGATGTACCAGAGCCAGC \\
\hline & & $\begin{array}{l}\text { target } \\
\text { duplication }\end{array}$ & $\begin{array}{cc}\text { 3'P } & \text { target } \\
\text { duplication }\end{array}$ \\
\hline
\end{tabular}

4) A22 amino acid $170 \quad$ CCA to TCA

5) $A 206 \quad$ amino acid $261 \quad$ TCG to TTG

6) $A 206 \quad$ amino acid $274 \quad$ CAG to TAG

7) A329 amino acid $301 \quad$ GIN

8) ML66 splice donor mutation - junction between exons 3 and 4

$\begin{array}{llll}\text { Canton-S } & \text { AG/gt } & \ldots & \text { tcag/AA } \\ \text { ML } 66 & \text { Ag/ } / & \ldots & \text { tcag/AA }\end{array}$

All hid alleles for which polymorphisms were identified by sequencing are shown $1(3) 05014$ is a P-element insertion, and $40 C$ and $8 D$ were generated by imprecise excision of this insertion. A22, A206, and A329 were generated by Abbott and Lengyel (1991). A206 and $A 329$ are derived from the same parental chromosome (ru $h$ th ri $e$ ). A22 was induced on a distinct parental chromosome (red $e$ ). ML66 was generated in this study on the st $e$ parental chromosome. The wild-type chromosomes sequences were derived from Canton-S and st $e$ flies. None of the polymorphisms identified in the hid mutants was shared with either wild-type chromosome.

line transformants expressing the 5A1 hid cDNA under the control of the $h s p 70$ heat shock promoter $(\mathrm{P}[\mathrm{w} ; \mathrm{hs}-$ hid]). When $\mathrm{H} 99$ embryos carrying the hs-hid construct were heat shocked, we observed very high levels of PCD within $2 \mathrm{hr}$ upon heat shock induction (Fig. 4). These deaths appeared to occur by apoptosis, as judged by both acridine orange staining and TUNEL. No significant induction of PCD was observed in transformants without a heat shock, or in control embryos that were heat shocked but lacked the hs-hid transgene (data not shown). Furthermore, ectopic expression of several developmental control genes under the $h s p 70$ promoter, such as hs-disco (A. Campos and H. Steller, unpubl.), hs-engrailed (Poole and Kornberg 1988), or hs-hedgehog (Tabata and Kornberg 1994), all failed to induce PCD in H99 embryos (data not shown). The only other construct that had a similar ability to induce apoptosis under these experimental conditions was a hs-rpr transgene (White et al. 1994; K. White and H. Steller, unpubl.). These results provide strong support for the idea that hid functions in PCD.

The hs-hid transgene also induces ectopic cell death in wild-type embryos following heat shock. The cell death induced by the hs-hid transgene is lethal to wildtype embryos, and a single heat pulse during embryogenesis kills all flies bearing the hs-hid construct (data not shown). We considered the possibility that the ability of hid to activate PCD is enhanced by the presence of $r p r$. To test this idea, the amount of $h s-h i d$ induced cell death in a wild-type and an H99/H99 genetic background was compared. No reproducible differences between $H 99$ and wild-type embryos were detected, indicating that the ability of hid to kill is not significantly augmented by the presence of an endogenous rpr gene. We also used in situ hybridization to examine whether hs-hid-induced apoptosis in a wild-type background would lead to rpr mRNA expression. This point deserved some attention, because rpr is normally expressed in all cells that will undergo apoptosis. However, the embryonic pattern of rpr mRNA was not significantly affected by ectopic expression of hid, even under conditions where large numbers of cells were dying (data not shown). We conclude that the induction of PCD by hid occurs independently of $r p r$. 

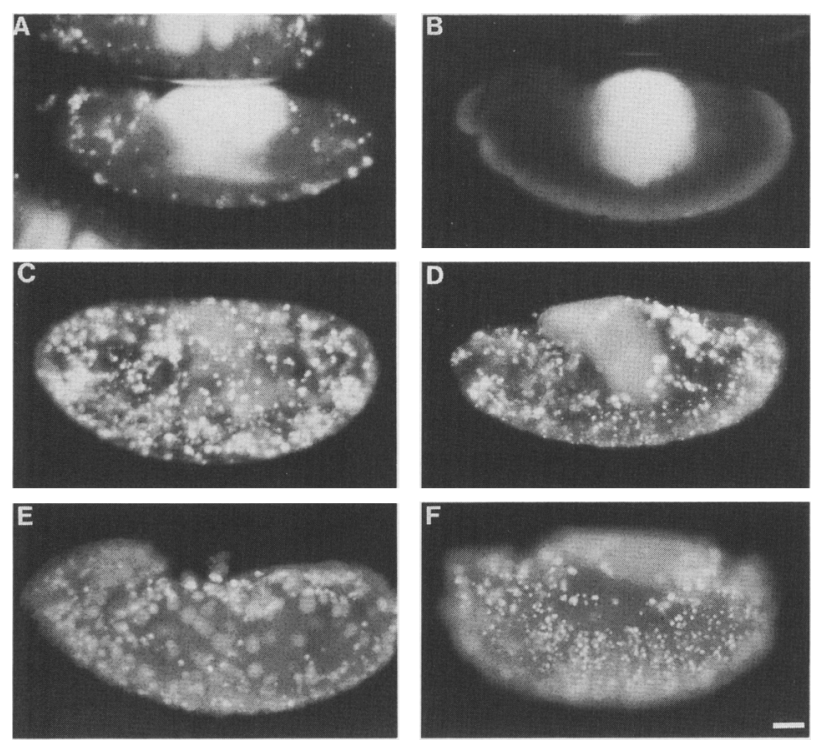

Figure 4. Patterns of cell death in heat-shocked wild-type, $H 99$, and hs-hid embryos. Lateral views of 10 - to 14 -hr-old embryos stained with acridine orange to visualize apoptosis. All embryos were subjected to an identical heat shock regimen /see Materials and methods). Each brightly staining small dot represents a dead or dying cell or cell fragment. $|A|$ Cell death in a heat shocked wild-type embryo. Following heat shock, the pattern of cell death in wild-type embryos is still characteristically patterned and virtually undistinguishable from the pattern of cell death seen in non-heat-shocked wild-type embryos. The large brightly stained area in the center of the embryo is the yolk. (B) Cell death in a heat-shocked $H 99$ embryo. Heat shock alone cannot induce cell death in the fully cell-death-defective $H 99$ mutant embryos. Other than the yolk, which can be seen to autofluoresce, there is a conspicuous lack of any acridine orange staining in this heat-shocked $H 99$ embryo. $(C-F)$ Embryos from a collection of either hs-hid4; H99/TM3 $(C, D)$ or hs-hid2; H99/ $T M 3\langle E, F\rangle$ flies. Following heat shock, all embryos carring the hs-hid transgene (here, examples from two independent transgenic lines are shown/ have significantly more cell death than wild type (cf. $A$ ). In addition, the heat-shocked hs-hid embryos display abnormal morphology and fail to develop properly. Equivalent levels of cell death are induced in both heat-shocked wild-type and heat-shocked $H 99$ mutant embryos when both carry the $h s-h i d$ transgene. Bar, $50 \mu \mathrm{m}$.

\section{hid $m R N A$ is expressed in many regions where cell death occurs}

The distribution of hid mRNA in embryos was determined using whole-mount in situ hybridization with digoxygenin-labeled RNA probes. We found that the pattern of hid expression is highly dynamic and complex throughout embryogenesis. Significantly, hid is expressed in many regions where cell death occurs (Fig. $5 \mathrm{~A}-\mathrm{F})$. For example, in stage 11 embryos, we found both acridine orange staining and hid mRNA hybridization in the head and gnathal segments, as well as segmentally repeated throughout the extended germ band (Fig. 5A,B). In slightly older embryos undergoing early stages of head involution, we observed a correspondence between the patterns of cell death and hid mRNA expression, partic- ularly in the head (Fig. 5C-F). We also found evidence of hid expression within macrophages, apparently confined to cell corpses that have been engulfed (Fig. 5G,H).

Although there is significant overlap between the patterns of hid expression and acridine orange staining, these patterns are not entirely coincident. For example, hid mRNA is found throughout the entire optic lobe primordium, but only some of these cells undergo apoptosis (Fig. 5E,F). It is possible that post-translational control mechanisms restrict the production of active Hid protein to a subset of cells expressing hid RNA. Alternatively, not all cells may be equally sensitive to the amount of hid expression (see Discussion). In addition, although there is considerable cell death in the ventral nerve cord during late embryogenesis (Abrams et al. 1993), little or no hid expression can be detected at this time (not shown). Perhaps hid is not required for these deaths; alternatively, hid may be expressed in the ventral nerve cord below our level of detection.

\section{Ectopic expression of hid driven by an eye-specific promoter results in eye ablation}

If hid expression is sufficient to induce PCD, it should be possible to use hid to kill tissues that normally live. To test this possibility we used a tissue-specific promoter to direct expression of hid to the developing Drosophila eye. The compound eye is particularly well suited for this type of study because it is a nonessential tissue, and its different cell types and their mode of development have been characterized extensively. The hid cDNA was placed under the control of an eye-specific regulatory element in the pGMR transformation vector /Hay et al. 1994), and the resulting construct, $p G M R-$ hid, was used to generate germ-line transformants.

Transformants carrying a single copy of $p G M R-h i d$ displayed a dramatic eye ablation phenotype (Fig. 6). Normally, compound eyes consist of $\sim 800$ regular units, called ommatidia, each of which consists of several distinct cell types (Fig. 6A). In pGMR-hid transformants, only undifferentiated cuticle and a dense band of bristles remained in the places normally occupied by the compound eyes (Fig. 6B). It appears that these bristles represent the mechanosensory bristles normally found at the corner of each ommatidium. Apparently, these cells are less susceptible to hid-induced death. However, the number of these cells is severely reduced in transformants that are homozygous for the $p G M R-h i d$ transgene, indicating that their survival is sensitive to the dosage of hid expression. It is possible that the hid transgene is expressed only weakly in bristle precursors. Alternatively, bristle cells may be better protected against hid-induced PCD. Interestingly, a very similar phenotype is obtained upon expression of $r p r$ in the developing retina (K. White and $H$. Steller, unpubl.). Significantly, the hid-induced eye phenotype is completely suppressed by coexpression of the baculovirus p35 gene (Fig. 6D), which has been shown previously to inhibit PCD (Hay et al. 1994; Clem and Miller 1994; Rabizadeh et al. 1993; Sugimoto et al. 1994). Flies expressing both hid and p35 

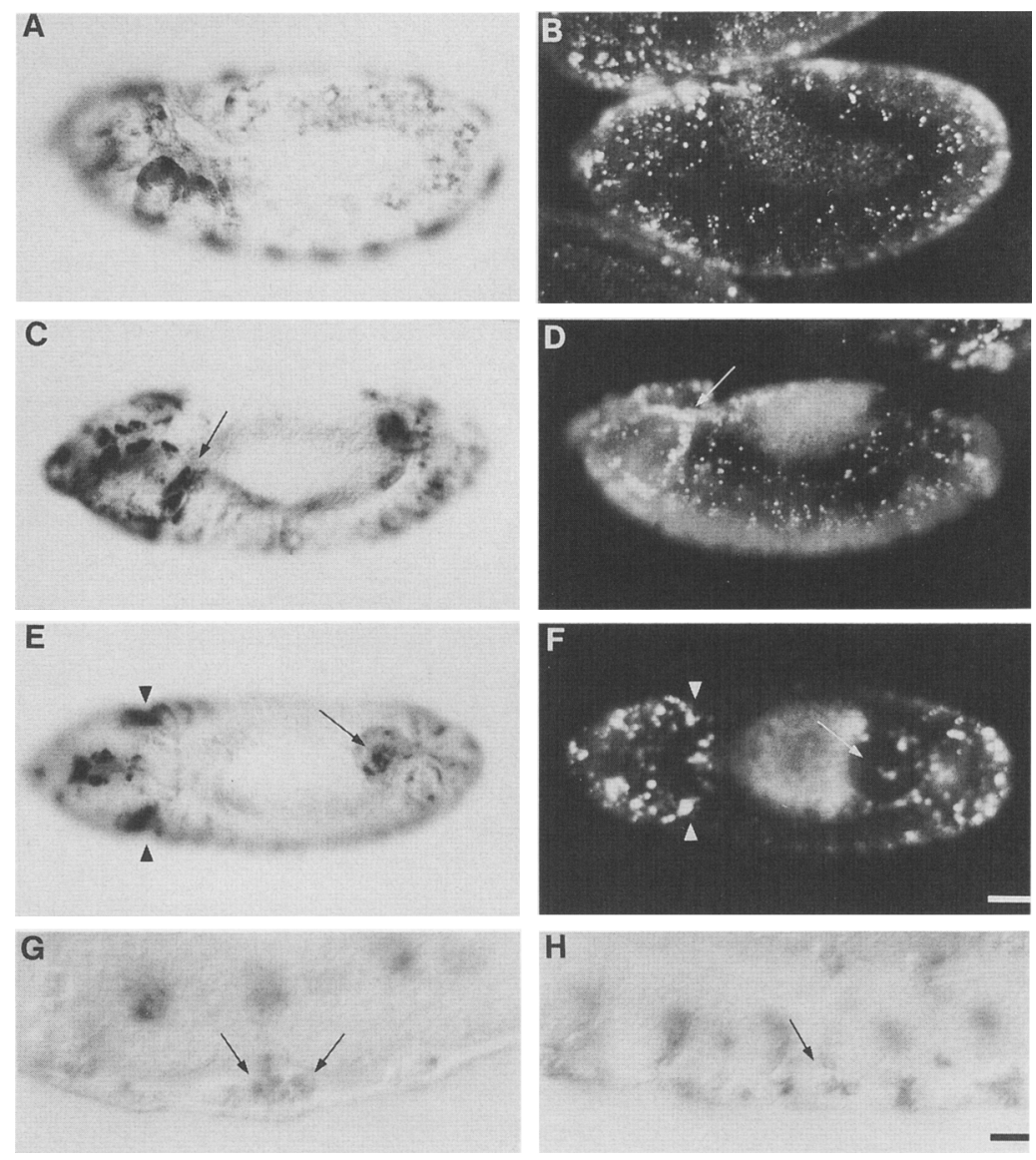

Figure 5. Comparison of the tissue distribution of hid mRNA and the pattern of PCD in the embryo. The hid transcript was detected in wholemount embryos by in situ hybridization with digoxigenin-labeled RNA probes $(A, C, E, G, H)$. Cell death was visualized by acridine orange staining $(B, D, F)$. Throughout all stages of embryonic development, there are many similarities among the patterns of hid mRNA expression and PCD. $(A, B)$ Lateral view of stage 11 embryos. hid mRNA and acridine orange staining are found in the head and gnathal segments, and are segmentally repeated throughout the extended germband. $(C, D)$ Stage 12 embryos. The correspondence between the pattern of hid mRNA expression and cell death is particularly striking in the head region, e.g., in the optic lobe region (arrow). $(E, F)$ Dorsal view of late stage 12 embryos. Both hid mRNA and cell death are found in the clypeolabrum, the invaginating optic lobe primordia (arrowheads), and a portion of the hindgut (arrow). $(G, H)$ hid expression is found in cell corpses engulfed by macrophages (arrows), indicating that at least some hid-expressing cells undergo $\mathrm{PCD}$ and are recognized by phagocytic cells. Bar, $(A-F) 50 \mu \mathrm{m} ;(G, H) 17 \mu \mathrm{m}$. in the retina have an eye morphology that is very similar to transformants for p35 alone (Fig. 6C). This indicates that hid expression in the retina leads specifically to the induction of PCD but otherwise does not interfere with eye development. This result also suggests that protective functions, like p35, operate genetically downstream of hid.

Ectopic expression of hid in the developing retina appears to have no detrimental effects outside the visual system. Transformants that are homozygous for the pGMR-hid transgene have no other detectable phenotypes and are viable and fertile. In addition, the ability of hid to kill appears to be cell autonomous, at least on a global level, because mosaic animals expressing hid in only a portion of the retina have morphologically normal sectors of the eye abutting the ablated portions (J. Agapite and H. Steller, unpubl.). This indicates that tissue-specific expression of hid may represent an efficient tool for the ablation of specific cell types in Drosophila. Finally, as discussed below, the dosage sensitivity of the eye phenotype in $p G M R$-hid transformants suggests a powerful new genetic screen for isolating cell death genes in Drosophila.

\section{Discussion}

hid is sufficient for the induction of $P C D$

Deletions of chromosomal region $75 \mathrm{C} 1,2$, including
H99, protect against the induction of PCD in response to many different death-inducing signals. Previous work has implicated one gene in this interval, rpr as a central control function for the induction of apoptosis in Drosophila (White et al. 1994). However, this earlier work did not exclude the possible existence of another cell death gene in this interval. We examined the DNA encompassed by the H99 deficiency for other transcription units that might also serve cell death functions. The results from this study strongly support a model in which the hid gene encodes another cell death function in $75 \mathrm{C} 1,2$ that acts at the same step in apoptosis as rpr. To our knowledge, hid and $r p r$ are the only cell death genes in the $\mathrm{H} 99$ interval.

Mutations in the hid gene lead to reduced levels of cell death and the presence of extra cells, most notably in the head region of the Drosophila embryo. hid mutant embryos also have a striking inability to retract the head into the thoracic region, a process called head involution (Abbott and Lengyel 1991). Because there is a considerable amount of cell death associated with the morphogenetic movements of head involution, it is possible that this head phenotype results from the decreased amount of cell death that we have observed in hid mutants. The hid phenotype has variable penetrance and some adult flies emerge. These rare survivors have other phenotypes, such as rotated genitalia and a wing defect (Abbott and Lengyel 1991) that may be attributable to a decrease 

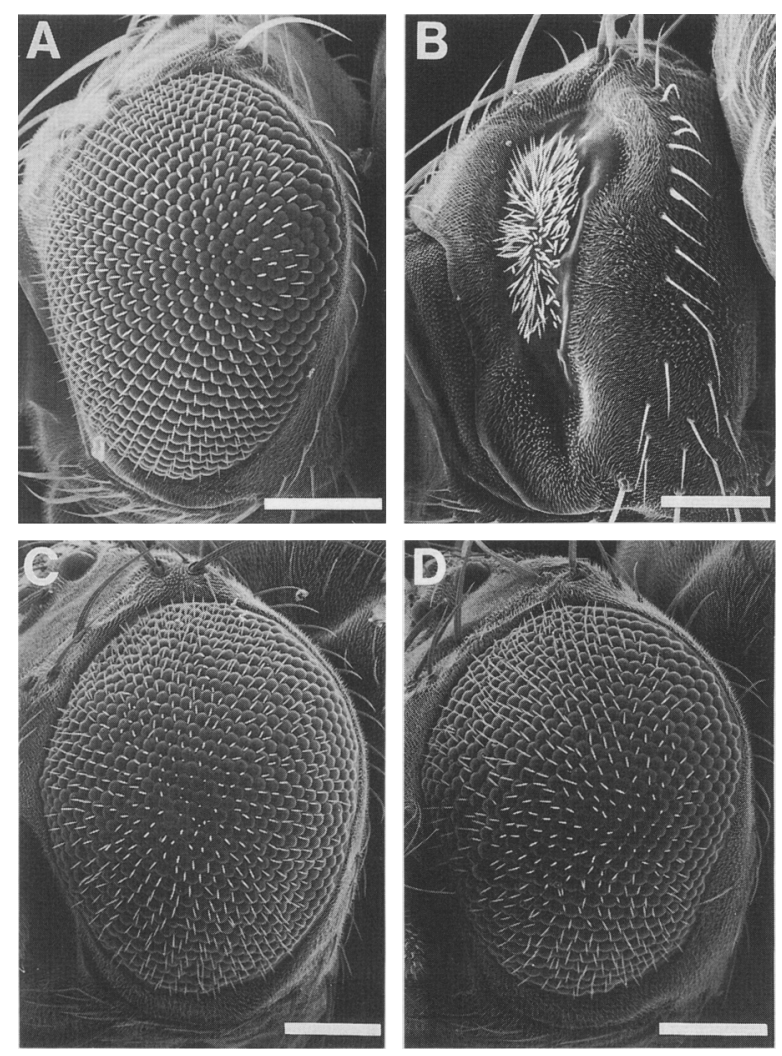

Figure 6. Scanning electron micrographs (SEMs) of the compound eye of wild-type and $p G M R-h i d$ flies. Expression of hid in the developing retina causes eye ablation, which is suppressed by coexpression of the anti-apoptotic p35 protein. SEMs of the compound eye of wild type $(A)$, pGMR-hid $/+(B)$, pGMR-p35/pGMR-p35 (C), and pGMR-hid/pGMR-p35 (D) flies are shown. In wild-type flies $(A)$, the compound eye is composed of regularly spaced ommatidia with mechanosensory bristles protruding from each ommatidium. In $p G M R-h i d$ transformants $(B)$, no ommatidial units are visible and undifferentiated cuticle and bristles fill the space normally occupied by ommatidia. These bristles are likely to be the mechanosensory bristles normally found at the corner of each ommatidium. The presence of a single copy of the pGMR-p35 transgene restores the ommatidia that are slightly disorganized $(D)$. A similar disorganization is seen in flies expressing p35 alone $\{C$, see also Hay et al. 1991), apparently because of the suppression of cell death that normally occurs during retinal development (Wolff and Ready 1991; Hay et al. 1994). Bar, $100 \mu \mathrm{m}$.

of PCD during imaginal development. The wings of hid adult flies of all ages are opaque and resemble the wings of newly eclosed wild-type adults (data not shown). In wild-type flies, immediately prior to and after eclosion, all of the cells in the wing intervein regions die and the wing flattens into what is essentially two cuticular sheets (Johnson and Milner 1987). It is possible that the wings of old hid flies remain opaque because of a failure of the intevein cells to undergo PCD.

Strong evidence for a role of hid in PCD comes from the observation that hid expression is sufficient to induce high levels of cell death in $\mathrm{H} 99$ embryos. Expression of a hid cDNA under the control of the heat-induc- ible $h s p 70$ heat shock promoter (hs-hid) leads to rapid and widespread induction of PCD in both wild-type and H99 embryos. There are two possible explanations for this result. First, hid may have a physiological role in the induction of apoptosis. Alternatively, the ectopic expression of hid could generate a developmental conflict that would subsequently activate the cell death program. However, there are strong reasons to believe that the latter explanation is highly unlikely. Embryos that are homozygous for the $\mathrm{H} 99$ deletion are virtually resistant to the induction of apoptosis by different death-inducing signals (White et al. 1994). Not only do normal developmental cell deaths fail to occur in H99 embryos, but ectopic cell deaths that are usually seen in many developmental mutants, such as crumbs, are blocked as well. In addition, $\mathrm{H} 99$ protects significantly against $\mathrm{PCD}$ induced by ionizing radiation, and the amount of apoptosis induced by hid far exceeds the levels that can be caused by X-rays. Therefore, we do not expect the misexpression of developmentally important genes to induce PCD in H99 embryos. This notion has received direct experimental support, as we did not detect any significant levels of PCD in H99 embryos upon ectopic expression of three such genes, engrailed, disco, and hedgehog. Furthermore, within the time course of our experiments, ectopic expression of these genes failed to induce ectopic PCD even in wild-type embryos. We conclude that the ability of hs-hid to induce PCD in these experiments reflects a direct and normal function of hid in apoptosis and is not attributable to indirect effects on development.

Because the H99 deletion includes the rpr gene, the ability of hid to kill in an $\mathrm{H} 99$ mutant background must be independent from rpr function. However, it remained possible that hid would activate rpr expression in wildtype embryos, and/or that rpr and hid act synergistically. Our results indicate that neither of these possibilities is correct. In situ hybridization experiments demonstrate that the pattern of $r p r$ mRNA is not significantly altered by ectopic hid expression. Therefore, rpr does not need to be expressed during the activation of PCD induced by hid. In contrast, rpr expression is turned on by every other death-inducing stimulus tested to date. We also see no synergism between rpr and hid, because the ability of hs-hid and $p G M R$-hid to kill is not significantly affected by the presence of an endogenous rpr gene or rpr transgenes (data not shown). This suggests that hid and $r p r$ encode functions that act in parallel to induce PCD.

We have also tested the ability of hid to kill cells that would normally survive by directing its expression to the developing retina. A single copy of a transgene that expresses hid during eye development results in essentially complete eye ablation. This phenotype is apparently attributable to the activation of PCD, because it can be completely suppressed by the coexpression of the antiapoptotic baculovirus $\mathrm{p} 35$ protein. Therefore, hid expression appears to have no significant detrimental effects on eye development as long as the induction of PCD is blocked. This result indicates that the hid-induced eye ablation is not a secondary consequence of a develop- 
mental conflict created by the misexpression of hid and provides strong evidence for a direct role of hid in the activation of PCD. The one cell type that largely escapes hid-mediated cell killing is the mechanosensory bristle of the compound eye. It is possible that these cells express relatively low levels of the transgene. Alternatively, these cells may either contain high levels of a negative regulator of PCD or low amounts of a cell death promoting factor acting downstream of hid. Interestingly, the survival of bristles appears to be sensitive to the dosage of hid, as their number is significantly reduced in animals containing two copies of the transgene. This dosage sensitivity may provide a powerful assay for identifying relatively subtle changes in the efficiency of PCD in Drosophila (see below).

The hid gene

Several lines of evidence confirm that we have molecularly identified the hid gene. The 05014 P-element insertion behaves as an allele of hid, and reversions of this allele were always associated with precise excision events. In contrast, imprecise excision of the P element did not restore hid function. The $05014 \mathrm{P}$ element is inserted within the longest ORF contained in the $4.2-\mathrm{kb}$ hid RNA, between amino acids 105 and 106 of the putative Hid protein. In addition, one chemically and two $\mathrm{X}$-ray-induced hid alleles were found to abolish or alter expression of the $4.2-\mathrm{kb}$ hid transcript. Finally, four hid alleles were associated with single-nucleotide changes in the Hid ORF (Table 1). Two of these mutations introduce stop codons and are predicted to result in premature translational termination of the Hid protein. Taken together, these results demonstrate that the ORF encoded at this locus is required for hid function.

Conceptual translation of the hid amino acid sequence reveals that hid encodes a novel 410 -amino-acid protein that is fairly basic and contains a high number of charged residues. There is no indication that hid is secreted, as it does not contain any obvious signal sequences. The expected intracellular localization of the Hid protein is also consistent with its apparent cell-autonomous function in PCD. Hid contains several potential phosphorylation sites, indicating that it may be subject to post-translational modification. Interestingly, we find some limited sequence similarity between the amino-termini of the Hid and Rpr proteins (Fig. 3C). Given the overall functional correspondence of both genes, it is possible that this small block of similarity between Hid and Rpr has mechanistic significance. Consistent with this idea is the observation that the amino terminus of Rpr has been highly conserved between different Drosophila species. Furthermore, the two hid alleles that contain stop codons at amino acids 274 and 300, respectively, are weak mutations, suggesting that a significant amount of hid function is encoded in the amino-terminal part of the protein.

\section{Models for the role of hid in apoptosis}

The decision of whether a cell lives or undergoes apoptosis is determined by the interplay of both death-pro- moting and death-protective functions. Current evidence suggests that most, if not all, animal cells constitutively express all of the proteins necessary to execute the cell death program (Raff 1993; Jacobson et al. 1994). However, it appears that the inadvertant activation of cell death machinery is prevented by negative regulators of cell death, such as ced-9/Bcl-2 family members /Vaux et al. 1988; Hengartner et al. 1992; Boise et al. 1993; Oltvai et al. 1993; Hengartner and Horvitz 1994c). What are the genes involved in triggering the activation of the apoptotic program? We have proposed previously that $r p r$ is a global regulator of PCD (White et al. 1994). The results from this study suggest that hid serves a similar function for the induction of PCD in Drosophila. We have strong reasons to believe that hid and rpr do not encode death effector proteins. When $\mathrm{H} 99$ embryos are irradiated with very high doses of X-rays, some cell death can be induced (White et al. 1994). Significantly, the morphology of the few cell deaths that are observed under these circumstances is indistinguishable from apoptosis seen in wild-type embryos. This suggests that the basic cell death machinery is present in $\mathrm{H} 99$ embryos but fails to be efficiently activated in the absence of hid and $r p r$.

The functions of hid and rpr appear to be at least partially redundant, as mutations in hid do not completely block PCD, and expression of either gene alone is sufficient to induce apoptosis in $\mathrm{H} 99$ embryos. The ability of hid to cause cell death in the absence of endogenous rpr and rpr to cause cell death in the absence of endogenous hid argues that neither gene acts through the other but, rather, that they act in parallel pathways or in parallel branches of a common pathway. Consistent with this idea, we did not find changes in the pattern of rpr mRNA expression in hid embryos (data not shown). Similarly, we could not detect changes in the pattern or levels of $r p r$ expression in hs-hid embryos following heat shock, nor did we find alterations in hid expression in hs-rpr embryos (data not shown). Finally, we have also been unable to obtain any evidence for synergism between rpr and hid in animals containing both the $h s-r p r$ and hs-hid or the $p G M R-r p r$ and $p G M R-h i d$ transgenes.

In the Drosophila embryo, hid RNA is generally found in regions where cell death occurs. However, despite these striking correlations, there are also clear discrepancies between the pattern of PCD and hid mRNA distribution. Overall, hid RNA expression tends to be more widespread than the amount of cell death normally observed. This is in contrast to rpr, which appears to be selectively expressed in all cells that are destined to undergo apoptosis. There are several possible explanations for this discrepancy. Most notably, the production of active Hid may be subject to post-transcriptional modifications, such as translational regulation or post-translational modifications. Alternatively, different cells may express different amounts of protective functions and may therefore vary in their sensitivity to the amount of hid expression required to induce PCD. Finally, it is possible that certain cells have insufficient amounts of death-promoting functions that act downstream from 
hid. In any case, it is clear that overexpression of hid can largely override any normally operating regulatory mechanisms, as shown by the increased levels of PCD in hs-hid embryos, and the ability of $p G M R-h i d$ to ablate the compound eye. In these instances, high levels of hid expression may conceivably titrate out protective functions or bypass the need for auxiliary factors. The biochemical mechanism by which hid activates apoptosis remains to determined. It is possible that Hid activates downstream targets that promote apoptosis, which may include ced-3/ICE or ced-4-like proteins /Yuan and Horvitz 1992; Yuan et al. 1993; Wang et al. 1994). Alternatively, Hid may block the action of negative regulators of cell death, such as Bcl-2/ced-9 family members. The fact that p35 expression can suppress hid-induced cell death indicates that hid acts genetically upstream of deathinhibiting functions like p35.

The ability of hid to rapidly induce apoptosis indicates that this gene may be used for targeted cell ablation in Drosophila. Methods developed previously that rely on the expression of toxin genes have found only limited use in Drosophila because of the considerable lag between toxin expression and cell killing, and because of the difficulties in restricting toxic effects to the targeted cells (Kunes and Steller 1991; Bellen et al. 1992; Moffat et al. 1992; discussed in Steller 1993). The use of hid instead of toxin genes may largely overcome these limitations. First, hid expression leads to the rapid onset of PCD, apparently as quickly as $1 \mathrm{hr}$. Second, it appears that hid needs to be expressed above a critical threshold to induce PCD, because we have not seen detrimental effects in $h s-h i d$ transformants raised at $29^{\circ} \mathrm{C}$. This threshold effect should greatly help restrict the specificity of ablation to the targeted cell type or tissue. By using the GAL4 system of Brand and Perrimon (1993), one may be able to ablate given tissues of interest with considerable facility. Because widespread expression of hid causes organismal lethality, the hs-hid transgene can also be used to select against offspring bearing specific chromosomes. Apparently, this method produces fewer escapers than when dominant temperature sensitive mutations are used for this purpose (L. Moore and R. Lehmann, pers. comm.).

Finally, $p G M R-h i d$ transformants may provide a powerful assay for identifying mutations in other cell death genes. Because the severity of the eye ablation phenotype is dosage-sensitive, relatively subtle changes in the level of $\mathrm{PCD}$ are expected to alter the eye phenotype. By screening for dominant genetic modifiers of $p G M R-h i d$, it should be possible to isolate mutations in cell death genes acting downstream of hid. A similar strategy has been highly successful for defining a genetic pathway for cell fate determination in the Drosophila eye (Simon et al. 1991; Gaul et al. 1992; Brunner et al. 1994). Therefore, we expect that the $p G M R$-hid transformants will greatly facilitate the elucidation of a genetic pathway for apoptosis in Drosophila. Because hid-induced cell death can be blocked by p35, and because p35 expression can protect against PCD in several other systems /Rabizadeh et al. 1993; Sugimoto et al. 1994; Clem and Miller 1994;
Hay et al. 1994), we expect that at least some of the genes in this pathway have been conserved in evolution.

\section{Materials and methods}

Drosophila stocks

$D f(3 L) H 99, h i d^{A 22}, h i d^{A 206}, h_{i d}^{A 329}, h_{i d}^{H 89}$, In(3L)hid ${ }^{\text {WR }+X 1}$, $h i d^{W R+E 1}$, and $h i d^{W R+E 4}$ were obtained from M. Abbott and J. Lengyel. 1(3)05014 (Karpen and Spradling 1992; Berkeley Drosophila Genome Project, pers. comm.) was provided by A. Spradling. hid ${ }^{4 O C}$ and $h i d^{8 D}$ were generated by excision of the $05014 \mathrm{P}$ element (see below). X14, X20, and X25 were generated by White et al. (1994). The wild-type strain used was Canton-S. All genetic symbols are described in Lindsley and Zimm (1992).

\section{Isolation of single gene mutations in 75C1,2}

Males of the genotype st $e$ were allowed to feed on a solution of $0.4 \mathrm{mg} / \mathrm{ml}$ of ENU with $1 \%$ sucrose for $24 \mathrm{hr}$. Mutagenized males were crossed en masse to virgin $S b H / T M 2$ females. Individual *st e/Sb $\mathrm{H}$ males were then crossed to WR4/TM3Sb females, and their progeny were scored for visible mutations uncovered by WR4 and for lack of *st $e / W R 4$ individuals, indicating the presence of a recessive lethal mutation when in combination with the WR 4 chromosome. Candidates for mutations in $75 \mathrm{Cl}, 2$ were then tested for lethality or segregation of the visible phenotype by crossing them to WR10 flies. Approximately 23,000 mutagenized third chromosomes were screened, and we identified five chromosomes that were lethal in combination with both WR4 and WR10. These mutations were determined to be hid alleles by standard complementation analysis. When placed in trans to existing hid alleles, significant lethality was observed. Some escapers were also recovered, which then displayed the characteristic adult hid phenotypes (Abbott and Lengyel 1991). st $e$ and $S b H / T M 2$ stocks were provided by R. Lehmann, Massachusetts Institute of Technology, Cambridge. $W R 4$ and $W R 10$ are genomic deficiencies that uncover $75 \mathrm{Cl}, 2$ (Segraves and Hogness 1990) and were provided by the Indiana Stock Center. The $H 99$ deficiency is entirely internal to the overlap defined by the deficiencies WR4 and WR1O (White et al. 1994).

\section{Excision of the 05014 insertion}

The 05014 P element was mobilized using $\Delta 2-3$ (99B), a genomic source of $\mathrm{P}$ transposase (Robertson et al. 1988). Excision chromosomes were identified by loss of the rosy ${ }^{+} \mathrm{ry}^{+}$marker. Reversion of the hid phenotype was scored by placing each excision chromosome in trans to H99. Forty-five percent of all excision events resulted in reversion of the hid phenotype.

\section{Histological methods}

Embryos were staged according to Campos-Ortega and Hartenstein (1985). Whole-mount in situ hybridizations and TUNEL/ in situ hybridization were performed as described in White et al. (1994). The probe used was generated by in vitro transcription of the antisense strand of the $5 \mathrm{Al}$ cDNA clone according to the protocol of the Boehringer RNA labeling kit. No hybridization with this probe was detected in embryos homozygous for $75 \mathrm{C} 1,2$ deficiencies. For TUNEL/hid mRNA double labelings, $W R+X 1 / H 99$ and Canton-S embryos were collected and aged until they were $8-13.5 \mathrm{hr}$ old at $25^{\circ} \mathrm{C}$ (stages 12-15). It is during this time that head involution occurs. The hid and Canton-S embryos were treated identically except that no hid mRNA 
probe was added to the Canton-S embryos. In a parallel experiment, Canton-S embryos were labeled with the hid probe to control for uniformity of labeling. Approximately $25 \%$ of the embryos from the hid egg collection did not label with the hid mRNA, confirming our expectation that these embryos were hid mutants. In contrast, $100 \%$ of Canton-S (wild-type) embryos taken through the in situ hybridization procedure in parallel did label with the hid mRNA. Because the amount of cell death in wild-type embryos changes considerably during development (Abrams et al. 1993), care was taken to ensure that patterns of cell death were compared between closely aged matched hid and wild-type embryos. Embryos were determined to be the same age by examining gut morphology, avoiding the problems associated with staging hid embryos by head morphology. To examine rpr expression in $W R+X 1$ embryos, pools of $W R+X 1 / H 99$ embryos were hybridized with both hid and $r p r$ mRNA probes. The patterns of hid and $r p r$ mRNA expression are sufficiently distinct to allow identification of embryos which hybridized with the rpr probe but not the hid probe. Those embryos were assumed to be genotypically hid and were examined for alterations in rpr expression as compared to wildtype embryos that had been probed with rpr alone.

Krüppel staining was done as described in Gaul et al. (1987). Embryos were aged to $12-17 \mathrm{hr}$ old (stages 15-17), by which time head involution is complete in wild-type embryos. hid embryos were identified as those in which head involution had failed. The alleles examined were $W R+X 1$ laverage number of cells per cluster $=13.6, n 42$ ) $A 206$ (average number of cells per cluster $=13.9, n=32$ ) $X 14 / H 99$ (average number of cells per cluster $=17.5, n=36$ ), and $W R+X 1 / H 99$ (average number of cells per cluster $=15.2, n=46$ ). Extra Krüppel-positive cells were also found in $W R+E 6, H 89$, and 05014/H99 embryos, but systematic large-scale counts were not done on these samples.

Acridine orange staining was performed as in Abrams et al. (1993).

\section{Identification of transcripts in $\mathrm{H} 99$}

Genomic DNA in the H99 interval was cloned by chromosomal walking using standard techniques (Sambrook et al. 1989). This DNA was probed with cDNA prepared from embryonic RNA to identify putative transcription units. Northern analysis was used to confirm the presence of transcribed sequences. Four transcripts were identified that gave reproducible Northern signals. One corresponded to $r p r$, and another to hid. The embryonic expression pattern of all four transcripts was determined by in situ hybridization.

\section{Plasmid rescue of genomic DNA flanking the 05014 insertion}

Molecular biological techniques were performed by standard procedures (Sambrook et al. 1989). Rescue of genomic sequences was performed basically as described in Steller and Pirrotta (1986). For plasmid rescue, genomic DNA from flies carrying the 05014 insertion was digested to completion with KpnI. Approximately $13 \mathrm{~kb}$ of genomic DNA flanking the site of in. sertion was recovered and mapped to a chromosomal walk in 75C (Segraves and Hogness 1990; Grether 1994) by Southern hybridization.

\section{Isolation and sequencing of the $5 A 1 \mathrm{cDNA}$}

The 8-kb EcoRI restriction fragment from phage 3506 (Segraves and Hogness 1990) was used to screen an eye disc cDNA library in $\lambda g t 10$ (constructed by A. Cowman). One clone, clone $5 \mathrm{Al}$ was isolated, which was longer than $2 \mathrm{~kb}$. The cDNA insert was subcloned into the EcoRI site of the pBluescript II SK ${ }^{+}$(Stratagene) cloning vector. Series of nested deletions for both strands of the cDNA were generated according to manufacturer's instructions using the Exo/Mung deletion kit (Stratagene). Sequencing of double-stranded DNA was carried out using Sequenase (U.S Biochemical Corp.) according to the manufacturer's instructions. Both strands were sequenced. Gaps in the series of nested deletions were filled by sequencing using oligonucleotide primers derived from the cDNA sequence determined previously. Determination of intron/exon boundaries and confirmation of the $5 \mathrm{Al}$ sequence were done by sequencing portions of phage clones corresponding to the genomic hid locus. Sequencing of the genomic hid locus was done using $\left[\gamma^{-}{ }^{33} \mathrm{P}\right]$ ATPlabeled oligonucleotide primers and the Circumvent sequencing kit (New England Biolabs) according to the manufacturer's instructions.

\section{Sequencing of the hid alleles}

Flies of each allele to be sequenced were crossed to $H 99$. When possible, DNA was isolated from adult flies of the genotype hid/H99. If no adults emerged from the cross, embryos with head involution defects were culled for DNA preparation. DNA was prepared as described in Ashburner (1989). Fragments corresponding to each coding exon of the hid locus were amplified by the polymerase chain reaction (PCR). The primers used for amplification are as follows: exon 1, 5'-GTGCCAACAAAGTGCA-3' and 5'-AATGGATATCCTGATTAACCCACAC-3'; exon 2,5 '-GATTTTCTTATTATGTGCCAACTGT-3' and 5'-TGATTTGATTTGCACCACCCAGGC-3'; exon 3, 5'-TCCAAGTCAACCGTCTATATGT- $3^{\prime}$ and $5^{\prime}$-TTGTGCATTC-

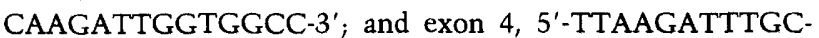
CTTCACGTGCACC-3' and 5'-AACTAAGTTTAGATCGG-3'. For each PCR reaction, $\sim 50 \mathrm{ng}$ of genomic DNA was used as template. A standard 50- $\mu$ l PCR reaction was set up according to protocols of Perkin-Elmer Cetus. Annealing temperatures for each set of primers are as follows: exon $1,52^{\circ} \mathrm{C}$; exon $2,56^{\circ} \mathrm{C}$; exon $3,60^{\circ} \mathrm{C}$; exon $4,50^{\circ} \mathrm{C}$. Cycling profiles for all amplifications were as follows: 1 cycle for $1 \mathrm{~min}$ at $94^{\circ} \mathrm{C}$, followed by 30 cycles of $1 \mathrm{~min}$ at $94^{\circ} \mathrm{C}, 2 \mathrm{~min}$ at the annealing temperature, 2 min $15 \mathrm{sec}$ at $72^{\circ} \mathrm{C}$ with $2 \mathrm{sec}$ added per cycle. This was followed by $10 \mathrm{~min}$ at $72^{\circ} \mathrm{C}$. Fifty percent of each reaction was loaded onto a $1 \%$ SeaPlaque GTG grade (FMC) low melting agarose gel in $1 \times$ TAE. Bands of the appropriate size were excised from the gel and diluted two- to threefold with water. Five microliters of this was used as sequencing template. Sequencing was done using $\left[\gamma^{-33} \mathrm{P}\right] \mathrm{ATP}$-labeled oligonucleotide primers and the Circumvent sequencing kit (New England Biolabs). For each polymorphism identified, a second, independent PCR reaction was performed and the allele resequenced.

Construction of the hs-hid transgene, genomic
transformation, and phenotypic analysis

A 450-bp BamHI-EcoRI fragment containing the Drosophila hsp 70 promoter from pUC hsB $\rightarrow \mathrm{R}$ ( $\mathrm{H}$. Steller) was cloned into the polylinker of pCaSpeR (Pirrotta 1988). An EcoRI fragment containing the hid cDNA was then cloned into the EcoRI fragment in the pCaSpeR/hsp 70 construct in the sense orientation. Orientation was determined using an assymetric restriction site in the hid cDNA. DNA used for transformation was purfied by the Qiagen plasmid purification kit and resuspended at a concentration of $1 \mathrm{mg} / \mathrm{ml}$. Cesium chloride-purified $\mathrm{p} \pi 25.7 \mathrm{wc}(\mathrm{Ka}$ ress and Rubin 1984) was used as the source of transposase and mixed with the plasmid DNA at a final concentration of 150 $\mu \mathrm{g} / \mathrm{ml}$. Embryos $(3090)$ were injected, and four independent 
transformed lines were isolated. hs-hid 1 and hs-hid 3 map to the third chromosome and hs-hid 2 and hs-hid 4 map to the $\mathrm{X}$. The $\mathrm{X}$ chromosome insertions were independently crossed in to the $H 99$ background. Embryos were collected at $22^{\circ} \mathrm{C}$ for $4-6 \mathrm{hr}$ from flies of the genotype $h s-h i d ; H 99 / T M 3 S b$. They were aged at $18^{\circ} \mathrm{C}$ until they were $8-12 \mathrm{hr}$ old and heat shocked in a water bath for $1 \mathrm{hr}$ at $39^{\circ} \mathrm{C}$. They were allowed to recover for $1 \mathrm{hr}$ at $25^{\circ} \mathrm{C}$ and then stained with acridine orange as in Abrams et al. (1993). Untransformed flies were also subjected to the same heat shock regimen; a few acridine orange-positive cells can be found in untransformed $\mathrm{H} 99$ embryos. Excess acridine orange staining was not observed in embryos that were not heat shocked.

To check that the effects observed were specific to hid expression and not to the transformation vector, or the heat shock promoter itself, three other heat shock constructs were tested: hs-disco (A. Campos and H. Steller, unpubl.), hs-engrailed (Poole and Kornberg 1988), and hs-hedgehog (Tabata and Kornberg 1994). These insertions were crossed independently into the $H 99$ background. For these experiments, embryos were collected from flies of the genotype hs-disco or hs-engrailed or hs-hedgehog/ ${ }^{+}, \mathrm{H} 9 \mathrm{I}^{+}$and subjected to the heat shock regimen described above to the $h s-h i d$ flies. No acridine orange staining was observed above background levels in flies bearing any of these constructs.

To check for evidence of synergism between hid and rpr, lines were constructed that contained either two copies of $h s-h i d$, two copies of $h s-r p r$, or one copy of each transgene (two transgenes total). Embryos were collected from these flies and subjected to the heat shock regimen described for the $h s$-hid experiment except that heat shocks were done at $32^{\circ} \mathrm{C}, 33^{\circ} \mathrm{C}$, and $37^{\circ} \mathrm{C}$ (in independent experiments). Embryos were stained with acridine orange and scored blind by two independent observers. No significant difference among the samples was observed.

\section{Construction of the pGMR-hid construct, genomic} transformation, and phenotypic analysis

A 3.9-kb EcoRI fragment containing the hid cDNA subclone 5AlB was inserted into the EcoRI cloning site of the pGMR vector (Hay et al. 1994) creating pGMR-hid.pGMR-hid and the helper plasmid p $25.7 \mathrm{wc}$ (Karess and Rubin 1984) were purified using the Qiagen plasmid purification kit. For transformation, pGMR-hid and p $\pi 25.7 \mathrm{wc}$ were mixed at a ratio of $10: 1$ such that the final total DNA concentration was $1 \mathrm{mg} / \mathrm{ml}$. $\mathrm{yw}^{67 \mathrm{c} 23}$ embryos (1800) were injected, and one transformed line was isolated in which $p G M R-h i d$ maps to the second chromosome. Scanning electron microscopy was performed on adult flies that were dehydrated through an ethanol series, critical point dried, and coated with gold-palladium.

\section{Acknowledgments}

We thank M. Abbott, R. Davis, B. Hay, H. Jäckle, T. Kornberg, G.M. Rubin, W.A. Segraves, A. Spradling, J. Tamkun, and the Berkeley Drosophila Genome Project for providing various reagents, information, and materials; L. Young for excellent technical assistance; and D. Stainier and M. Brodsky for comments on the manuscript. This work was supported in part by a Pew Scholars Award (H.S.), a National Institutes of Health postdoctoral fellowship (5 F32 NS08536) to K.W., and a postdoctoral fellowship from the American Cancer Society (J.M.A.). M.E.G., K.W., and J.M.A. were postdoctoral associates with and H.S. is an Associate Investigator with the Howard Hughes Medical Institute.

The publication costs of this article were defrayed in part by payment of page charges. This article must therefore be hereby marked "advertisement" in accordance with 18 USC section 1734 solely to indicate this fact.

\section{Note added in proof}

The sequence data for hid have been deposited in the GenBank data library under accession no. U31226.

\section{References}

Abbott, M.K. and J.A. Lengyel. 1991. Embryonic head involution and rotation of male terminalia require the Drosophila locus head involution defective. Genetics 129: 783-789.

Abrams, J.M., K. White, L.I. Fessler, and H. Steller. 1993. Programmed cell death during Drosophila embryogenesis. Development 117: 29-43.

Ashburner, M. 1989. Drosophila: A laboratory manual. Cold Spring Harbor Laboratory Press, Cold Spring Harbor, New York.

Bellen, H.J., D. D'Evelyn, M. Harvey, and S.J. Elledge. 1992. Isolation of temperature-sensitive diptheria toxins in yeast and their effects of Drosophila cells. Development 114: 787796.

Boise, L.H., M. Gonzales-Garcia, C.E. Postema, L. Ding, T. Lindsten, L.A. Turka, X. Mao, G. Nunez, and C.B. Thompson. 1993. $b c l \cdot x$, a $b c l-2$ related gene that functions as a dominant regulator of apoptotic cell death. Cell 74: 597-608.

Bonini, N.M., W.M. Leiseron, and S. Benzer. 1993. The eyes absent gene: Genetic control of cell survival and differentiation in the developing Drosophila eye. Cell 72: 379-395.

Brand, A.H. and N. Perrimon. 1993. Targeted gene expression as a means of altering cell fates and generating dominant phenotypes. Development 118: 401-415.

Brunner, D., N. Oellers, J. Szabad, W.H. Biggs III, S.L. Zipursky, and E. Hafen. 1994. A gain-of-function mutation in Drosophila MAP kinase activates multiple receptor tyrosine kinase signaling pathways. Cell 76: 875-888.

Bryant, P.J. 1988. Localized cell death caused by mutations in a Drosophila gene coding for a transforming growth factor- $\beta$ homolog. Dev. Biol. 128: 386-395.

Campos, A.R., K.-F. Fischbach, and H. Steller. 1992. Survival of photoreceptor neurons in the compound eye of Drosophlia depends on connections with the optic ganglia. Development 114: 355-366.

Campos-Ortega, J.A. and V. Hartenstein. 1985. The embryonic development of Drosophila melanogaster. Springer-Verlag, New York.

Cheyette, B.N.R., P.J. Green, K. Martin, H. Garren, V. Hartenstein, and S.L. Zipursky. 1994. The Drosophila sine oculis locus encodes a homeodomain-containing protein required for the development of the entire visual system. Neuron 12: 977-996.

Clem, R.J. and L.K. Miller. 1994. Induction and inhibition of apoptosis by insect viruses. In Apoptosis II. (ed. L.D. Tomei and F.O. Cope), pp. 89-110. Cold Spring Harbor Laboratory Press, Cold Spring Harbor, New York.

Ellis, R.E., J. Yuan, and H.R. Horvitz. 1991. Mechanisms and functions of cell death. Annu. Rev. Cell Biol. 7: 663-698.

Fischbach, K.-F. and G. Technau. 1984. Cell degeneration in the developing optic lobes of the sine oculis and small-opticlobes mutants of Drosophila melanogaster. Dev. Biol. 104: 219-239.

Fristrom, D. 1969. Cellular degeneration in the production of some mutant phenotypes in Drosophila melanogaster. Development 120: 2121-2129.

Gaul, U., E. Siefert, R. Schuh, and H. Jäckle. 1987. Analysis of Krüppel protein distribution during early Drosophila devel- 
opment reveals posttranscriptional regulation. Cell 50: 639647.

Gaul, U., G. Mardon, and G.M. Rubin. 1992. A putative Ras GTPase activating protein acts as a negative regulator of signaling by the Sevenless receptor tyrosine kinase. Cell 68: 1007-1019.

Gavrieli, Y., Y. Sherman, and S.A. Ben-Sasson. 1992. Identification of programmed cell death in situ via specific labeling of nuclear DNA fragmentation. $J$. Cell Biol. 119: 493-501.

Grether, M.E. 1994. "Molecular genetic analysis of larval visual system development and programmed cell death in Drosophila." Ph.D. thesis, Massachusetts Institute of Technology, Cambridge, MA.

Hay, B.A., T. Wolff, and G.M. Rubin. 1994. Expression of baculovirus P35 prevents cell death in Drosophila. Development 120: 2121-2129.

Hengartner, M.O. and H.R. Horvitz. 1994a. Programmed cell death in Caenorhabditis elegans. Curr. Opin. Genet. Dev. 4: $581-586$.

. 1994b. The ins and outs of programmed cell death during C. elegans development. Phil. Trans. R. Soc. (Lond.) B Biol. Sci. 345:243-246.

$-1994 \mathrm{c}$. The C. elegans cell survival gene ced-9 encodes a functional homolog of the mammalian proto-oncogene $b c l$ 2. Cell 76: 665-676.

Hengartner, M.O., R.E. Ellis, and H.R. Horvitz. 1992. C. elegans gene ced-9 protects cells from programmed cell death. $\mathrm{Na}$ ture 356: 494-499.

Jacobson, M.D., J.F. Burne, and M.C. Raff. 1994. Programmed cell death and Bcl-2 protection in the absence of a nucleus. EMBO I. 13: 1899-1910.

Johnson, S.A. and M.J. Milner. 1987. The final stages of wing development in Drosophila melanogaster. Tissue \& Cell 19: $505-513$.

Karess, R.E. and G.M. Rubin. 1984. Analysis of P transposable element functions in Drosophila. Cell 38: 135-146.

Karpen, G.H. and A.C. Spradling 1992. Analysis of subtelomeric heterochromatin in the Drosophila minichromosome Dp1 187 by single P-element insertional mutagenesis. Genetics 132: 737-753.

Kerr, J.F.R., A.H. Wyllie, and A.R. Currie. 1972. Apoptosis: A basic biological phenomenon with wide ranging implications in tissue kinetics. Br. J. Cancer 26: 239-257.

Kimura, K. and J.W. Truman. 1990. Postmetamorphic cell death in the nervous and muscular systems of Drosophila melanogaster. I. Neurosci. 10: 403-411.

Kunes, S. and H. Steller. 1991. Ablation of Drosophila photoreceptor cells by conditional expression of a toxin gene. Genes \& Dev. 5: 970-983.

Lindsley, D.L. and G.G. Zimm. 1992. The genome of Drosophila melanogaster. Academic Press, San Diego, CA.

Magrassi, L. and P.A. Lawrence. 1988. The pattern of cell death in fushi tarazu, a segmentation gene of Drosophila. Development 104: 447-451.

Meyertholen, E.P., P.J. Stein, M.A. Willams, and S.E. Ostroy. 1987. Studies of the Drosophila norpA phototransduction mutant. II. Photoreceptor degeneration and rhodopsin maintenance. J. Comp. Physiol. 161: 793-798.

Moffat, K.G., H.H. Gould, H.K. Smith, and C.J. O'Kane. 1992. Inducible cell ablation in Drosophila by cold-sensitive ricin A chain. Development 114: 681 .

Oltavi, Z.N., C.L. Milliman, and S.J. Korsmeyer. 1993. Bcll-2 heterodimerizes in vivo with a conserved homlog, Bax, that accelerates programmed cell death. Cell 74: 609-619.

Patel, N.H., E. Martin-Blanco, K.G. Coleman, S.J. Poole, M.C. Ellis, T.B. Kornberg, and C.S. Goodman. 1989. Expression of engrailed proteins in arthropods, annelids, and chordates. Cell 58: 955-968.

Pirrotta, V. 1988. Vectors for P-element transformation in Drosophila. In Vectors. A survey of molecular cloning vectors and their uses. (ed. R.L. Rodriguez and D.T. Denhardt), pp. 437-456. Butterworths, Boston, MA.

Poole, S.J. and T. Kornberg. 1988. Modifying expression of the engrailed gene of Drosophila melanogaster. Mechanisms of segmentation. Development (Suppl.) 104: 85-93.

Rabizadeh, S., D.J. La Count, P.D. Friesen, and D.E. Bredesen. 1993. Expression of the baculovirus p35 gene inhibits mammalian cell death. J. Neurochem. 61: 2318-2321.

Raff, M.C., B.Z. Barres, J.F. Burne, H.S. Coles, Y. Ishizaki, and M.D. Jacobson. 1993. Programmed cell death and the control of cell survival: Lessons from the nervous system. Science 262: 695-700.

Robertson, H.M., C.R. Preston, R.W. Phillis, D.M. JohnsonSchlitz, W.K. Benz, and W.R. Engels. 1988. A stabel source of P-element transposase in Drosophila melanogaster. Genetics 118: 6341-6351.

Robinow, S., W.S. Talbot, D.S. Hogness, and J.W. Truman. 1993. Programmed cell death in the Drosophila CNS is ecdysoneregulated and coupled with a specific ecdysone receptor isoform. Development 119: 1251-1259.

Sambrook, J., E.F. Fritsch, and T. Maniatis. 1989. Molecular cloning: A laboratory manual. Cold Spring Harbor Laboratory Press, Cold Spring Harbor, New York.

Segraves, W.A. and D.S. Hogness. 1990. The E75 ecdysone-inducible gene responsible for the 75B early puff in Drosophila encodes two new members of the steroid receptor superfamily. Genes \& Dev. 4: 204-219.

Simon, M.A., D.D.L. Bowtell, G.S. Dodson, T.R. Laverty, and G.M. Rubin. 1991. Rasl and a putative guanine nucleotide exchange factor perform crucial steps in signaling by the Sevenless protein tyrosine kinase. Cell 67: 701-716.

Steller, H. 1993. Toxin ablation in Drosophila. In Cellular interactions in development (ed. D.A. Hartley), pp. 77-92, Oxford University Press, Oxford, UK.

Steller, H. and M. Grether. 1994. Programmed cell death in Drosophila. Neuron 13: 1269-1270.

Steller, H. and V. Pirrotta. 1986. P transposons controlled by the heat shock promoter. Mol. Cell. Biol. 6: 1640-1649.

Steller, H., K.-F. Fischbach, and G.M. Rubin. 1987. disconnected: A locus required for neuronal pathway formation in the visual system of Drosophila. Cell 50: 1139-1153.

Steller, H., J.M. Abrams, M.E. Grether, and K. White. 1994. Programmed cell death in Drosophila. Proc. R. Soc. (Lond.) B Biol. Sci. 345: 247-250.

Sugimoto, A., P.D. Friesen, and J.H. Rothman. 1994. Baculovirus $\mathrm{p} 35$ prevents developmentally regulated cell death and rescues a ced- 9 mutant in the nematode Caenorhabditis elegans. EMBO /. 13: 2023-2028.

Tabata, T. and T.B. Kornberg. 1994. Hedgehog is a signaling protein with a key role in patterning Drosophila imaginal discs. Cell 76: 89-102.

Truman, J.W., R.S. Thorn, and S. Robinow. 1992. Programmed neuronal death in insect development. I. Neurobiol. 23: 1295-1311.

Vaux, D.L., S. Cory, and J.M. Adams. 1988. bcl-2 gene promotes hemopoietic cell survival and cooperates with c-myc to immortalize pre-B cells. Nature 335: 440-442.

Vaux, D.L., I.L. Weissman, and S.K. Kim. 1992. Prevention of programmed cell death in Caenorhabditis elegans by human bcl-2. Science 258: 1955-1957.

Wang, L., M. Miura, L. Bergeron, H. Zhu, and J. Yuan. 1994. Ich-1, an ICE/ced-3-related gene encodes both positive and 
Grether et al.

negative regulators of programmed cell death. Cell 78: 739750.

White, K., M.E. Grether, J.M. Abrams, L. Young, K. Farrell, and H. Steller. 1994. Genetic control of programmed cell death in Drosophila. Science 264: 677-683.

Wolff, T. and D.F. Ready. 1991. Cell death in normal and rough eye mutants of Drosophila. Development 113: 825839.

Wyllie, A.H., J.F.R. Kerr, and A.R. Currie. 1980. Cell death: The significance of apoptosis. Int. Rev. Cytol. 68: 251-306.

Yuan, J. and H.R. Horvitz. 1992. The Caenorhabditis elegans cell death gene ced-4 encodes a novel protein and is expressed during the period of extensive programmed cell death. Development 116: 309-320.

Yuan, J., S. Shaham, H.M. Ellis, and H.R. Horvitz. 1993. The $C$. elegans cell death gene ced-3 encodes a protein similar to mammalian interleukin-1 $\beta$-converting enzyme. Cell 75: $641-652$. 


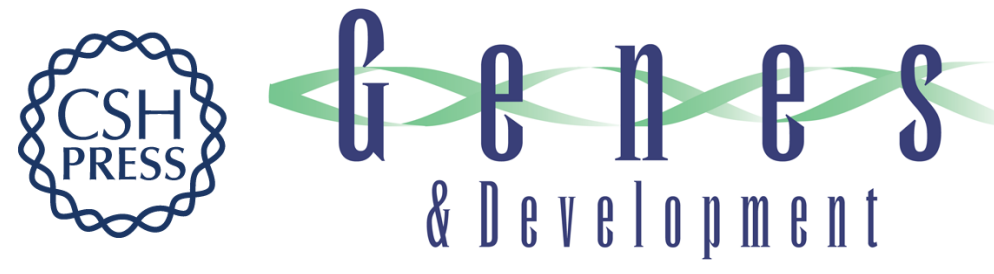

\section{The head involution defective gene of Drosophila melanogaster functions in programmed cell death.}

M E Grether, J M Abrams, J Agapite, et al.

Genes Dev. 1995, 9:

Access the most recent version at doi:10.1101/gad.9.14.1694

References This article cites 55 articles, 21 of which can be accessed free at:

http://genesdev.cshlp.org/content/9/14/1694.full.html\#ref-list-1

License

Email Alerting

Service

Receive free email alerts when new articles cite this article - sign up in the box at the top right corner of the article or click here.

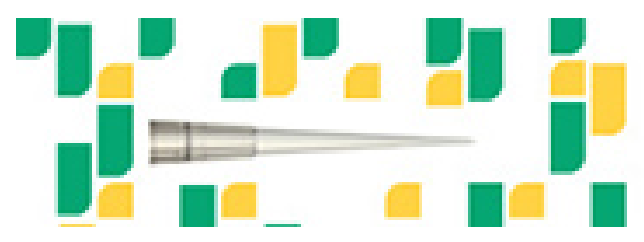

Focused on your science.

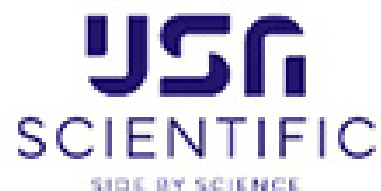

Copyright (c) Cold Spring Harbor Laboratory Press 